\title{
Teil VI: Berufliche Perspektive: UnVergessen als Türöffner
}

\author{
Katrin Bente Karl und Studierende
}

\section{Einleitung}

Katrin Bente Karl

Den abschließenden Teil bildet eine Darstellung dessen, inwiefern die Teilnahme am Projekt für zwei Studentinnen der Wegbereiter für ihren beruflichen Werdegang war. Hier berichtet zunächst Yvonne Behrens, eine Teilnehmerin der ersten Runde und aus diesem Buch bereits bekannte Wegbegleiterin des Projekts, von den tiefgreifenden Erfahrungen, die sie dazu bewegt haben, das Themengebiet zu ihrem Dissertationsthema zu machen. Aus diesem Interesse heraus erhielt sie eine Stelle als wissenschaftliche Mitarbeiterin (im Projekt UnVergessen), was wiederum der Türöffner für ihre Nachfolgestelle als wissenschaftliche Mitarbeiterin der FOM Hochschule für Oekonomie \& Management am ifgs Institut für Gesundheit \& Soziales war.

Im zweiten Abschnitt führt eine weitere ehemalige Teilnehmerin - Dorothea Laszczak, die ebenfalls in diesem Buch bereits zu Wort kam - aus, an welchen Punkten ihres Lebens Querverweise zur Pflege zu finden sind, und wie sie diese auf ihrem Weg in die berufliche Welt geprägt haben. Hier legt sie einen besonderen Schwerpunkt auf die nachhaltigen Eindrücke, die sie aus ihrer Masterarbeit mitgenommen hat. Diese hat sie aus ihrer Teilnahme an UnVergessen heraus entwickelt. Die tiefführende Auseinandersetzung mit den Biografien und Identitäten zweier Frauen mit polnischem Hintergrund schwingt noch immer in ihr nach

K. B. Karl und Studierende ( $\square)$

Seminar für Slavistik/Lotman-Institut, Ruhr-Universität Bochum, Bochum, Deutschland E-Mail: katrin.karl@ rub.de 
und leitet sie in ihrer Tätigkeit: Sie hat in Polen eine Vermittlungsagentur für Pflegekräfte ins Leben gerufen.

Um den Blick in die Zukunft zu werfen und damit auch zum Abschluss dieses Bandes zu kommen, sollen Ideen und Ansätze aus Sicht der Projektleitung skizziert werden, welche Richtungen in Zukunft eingeschlagen werden können und möglich erscheinen.

\section{Verbindung von Wissenschaft und Praxis zur Verbesserung der gesundheitlichen und sozialen Versorgung}

\section{Yvonne Behrens}

„Vergessen, Verwirrung, Veränderung: Was bleibt ist ein Mensch!“ (Tepper, 2012). Die Unterstützung von Menschen mit Demenz ist mir bereits seit meiner Jugend ein großes Bedürfnis. So unterstützte ich mehrsprachige demenziell erkrankte Personen in einer Pflegeeinrichtung durch den Einsatz meiner Polnischkenntnisse. Sowohl polnischsprachige Gespräche mit den Pflegebedürftigen als auch Gespräche mit den Pflegekräften über sprachbezogene Themen nutzte ich, um den Alltag der Pflegebedürftigen zu verschönern. Im Mittelpunkt stand hierbei der Mensch, nicht die Krankheit.

Das Projekt UnVergessen zeigte mir im Studium, dass man Menschen mit Demenz nicht nur sozial, sondern auch durch sprach- und sozialwissenschaftliche Forschung unterstützen kann. So kam ich zu meinem Promotionsthema „Rezeptiver und produktiver Wortschatz polnisch-deutscher bilingualer Demenzerkrankter", in welchem ich das Verständnis und die Sprachproduktion von monolingualen und bilingualen Menschen mit Demenz untersuche.

Nach meinem Master-Abschluss der Slavistik an der Ruhr-Universität Bochum habe ich als wissenschaftliche Mitarbeiterin im Projekt UnVergessen ${ }^{\text {PLUS }}$ (gefördert durch inSTUDIES ${ }^{\text {PLUS }}$ ) gearbeitet. Unter anderem übernahm ich die Koordination des Projekts und war hierbei für Akquise, Kontaktaufbau und anschlieBende Betreuung der teilnehmenden Pflegeeinrichtungen zuständig. Auf diese Art baute ich das Netzwerk für UnVergessen ${ }^{\text {PLUS }}$ und gleichzeitig für mein Promotionsprojekt aus. Des Weiteren arbeitete ich die wissenschaftlichen Erkenntnisse des Projekts auf und vertrat es auf unterschiedlichen Tagungen. Weiter bestand im Rahmen des Projekts die Möglichkeit, mehrsprachige Studierende und Pflegebedürftige zusammenzubringen. Hierbei ergaben sich besondere Momente für mich, da ich mehrere erste Treffen zwischen den Teilnehmenden begleiten konnte. Unvergessen bleibt das erste Treffen zwischen einem chinesischen Studierenden 
und einem Pflegebedürftigen, der seit langer Zeit die Möglichkeit eines Gesprächs auf Chinesisch bekam.

Seit dem Jahr 2019 arbeite ich an der FOM Hochschule für Oekonomie \& Management gGmbH am ifgs Institut für Gesundheit \& Soziales in den Projekten DigiCare country ${ }^{1}$, Research Network International ${ }^{2}$ und Com.HeNet ${ }^{3}$. Es handelt sich um internationale interdisziplinäre Forschungsprojekte, die ebenfalls die Thematik Gesundheit und teilweise auch das Thema Demenz fokussieren. Die Erfahrungen rund um das Thema Netzwerkausbau sowie den wissenschaftlichen Umgang mit vulnerablen Gruppen aus dem Projekt UnVergessen kann ich in meinem beruflichen Umfeld sehr gut einbringen und weiter ausbauen. Der wissenschaftliche Ansatz zur Verbesserung der Lebensqualität von einzelnen Menschen wird nun zwar auf größere Gruppen übertragen, die Idee bleibt jedoch die gleiche: Wissenschaft und Praxis verbinden! Dies war mir bereits im Projekt UnVergessen wichtig und bleibt auch weiterhin mein Ansatz, um gesellschaftliche Probleme anzugehen.

In allen vier Projekten wird wissenschaftliche Forschung mit der Praxis verbunden. Diese Verknüpfung hat zum einen Auswirkungen auf die Forschungsergebnisse der jeweiligen Kohorten, zum anderen erhöht es den Erfolg auf eine Übertragung in den Alltag: denn so können gesellschaftliche Probleme reduziert und das Wohlergehen der Bevölkerung gesteigert werden. Für mich stellt UnVergessen eben diese Verbindung dar, wodurch vulnerable Gruppen unterstützt werden. Dies leitet mich auch weiterhin in meiner beruflichen Tätigkeit.

\section{UnVergessen als Türöffner für den Weg in die Welt der Pflegevermittlung}

\section{Dorothea Laszczak}

UnVergessen, das Projekt ist tatsächlich unvergessen geblieben und hat einen großen Einfluss auf weitere Entwicklungen in meinem Leben ausgeübt. Nicht nur privat hatte das Projekt einen Einfluss auf mich. Wie sich auch mit der Zeit

\footnotetext{
${ }^{1}$ gefördert durch das Bundesministerium für Bildung und Forschung unter dem Förderkennzeichen 01DS19034.

${ }^{2}$ gefördert durch das Bundesministerium für Bildung und Forschung unter dem Förderkennzeichen 57509496.

${ }^{3}$ gefördert durch das ERASMUS+Programm der Europäischen Union unter der Projektnummer 20191 DE01 KA203 005025.
} 
nach meiner Teilnahme am Projekt herausstellte, brachte das Projekt in beruflicher Sphäre Perspektiven hervor.

Gerne möchte ich hier die Gelegenheit ergreifen und mich dazu äußern, wie sehr die Altenpflege mich mein Leben lang begleitet hat. Aufgewachsen bin ich in einer großen Familie. Als meine Eltern in den 1990-er Jahren aus Polen nach Deutschland kamen, arbeiteten beide in der Altenpflege. Auch meine drei Geschwister folgten beruflich unseren Eltern und absolvierten eine Ausbildung zum Altenpfleger/zur Altenpflegerin. Ich war die Einzige, die ein Studium aufnahm, welches keinen medizinischen Hintergrund hatte. Als ich nach dem Abitur das Studium der Slavischen Philologie und Sinologie aufnahm, dachte ich zu diesem Zeitpunkt noch nicht, dass die Altenpflege in meinem Leben wohl doch eine wichtige Rolle spielen würde.

Ich erinnere mich daran, dass, als ich von dem Projekt erfuhr, der sofortige Wunsch da war ein Teil dieses Projektes zu werden. Unsere Gruppe hatte eine einzigartige Energie, jeder von uns hat einen großartigen Teil zum Projekt beigetragen. Jedes unserer Treffen war spannend, jedes war emotional, jedes verging schnell und nach jedem freuten wir uns auf das nächste Treffen. Es ist ein Herzensprojekt, welches vom Menschen für den Menschen entstanden ist.

Als mein Studium sich langsam dem Ende näherte war es für mich eindeutig, dass ich meine Masterthesis mit dem Projekt verbinden möchte und so entstand ein wichtiger Beitrag zum Thema „Sprache und Identität im Kontext der Mehrsprachigkeit am Beispiel polnischsprachiger älterer Migrantinnen mit und ohne Demenz", durch den ich meine Kenntnisse und Erfahrungen in diesem Bereich noch vertiefen konnte und von dem ich auch heute noch profitiere.

In meiner Masterarbeit habe ich biografische Gespräche mit zwei pflegebedürftigen Migrantinnen mit polnischem Sprach- und Kulturhintergrund als Fallbeispiele näher beleuchtet. Die Zahlen der deutschen Polonia und die Alterung der Gesellschaft zeigen, wie relevant diese Gruppe bereits momentan ist und in der Zukunft sein wird. Eine der Frauen war an Demenz im mittleren Stadium erkrankt, die andere nicht. In meiner Arbeit lag der Fokus auf der Korrelation von Identitätskonstruktionen und der Zweisprachigkeit.

In den beiden untersuchten Fällen von Frau Pfeifer und Frau Konrad ${ }^{4}$ (demenziell verändert) standen sich zwei Leben gegenüber, die einige Parallelen, aber auch diverse Unterschiede implementierten. Die unterschiedlichen Migrationserfahrungen und -erinnerungen der beiden Erzählerinnen haben ohne Zweifel eine große Bedeutung im eigenen Identitätsverständnis gehabt. Als der signifikanteste Unterschied (neben der demenziellen Erkrankung) können die unterschiedlichen

\footnotetext{
${ }^{4}$ Die Namen wurden geändert.
} 
Erstsprachen der Sozialisation angesehen werden: die deutsche Sprache bei Frau Pfeifer und die polnische Sprache bei Frau Konrad. Diese Tatsache beeinflusste auch die Sprachwahl der beiden Frauen, da diese mit der Identität in enger Verbindung steht (Krappmann, 2000, S. 11). Da die Zweitsprache bei beiden Frauen später im Leben erworben wurde, gab sie nicht im selben Umfang wie die Erstsprache tiefverwurzelte und unbewusste Emotionen wieder. Ihren eigenen Selbsteinschätzungen zufolge wiesen die Probandinnen keine ausbalancierte Sprachkompetenz auf, es bestand somit ein subjektiv wahrgenommenes Ungleichgewicht zwischen der polnischen und deutschen Sprache. Für beide Frauen war die Zuordnung zu einer ethnischen Identität damit verbunden: Frau Pfeifer sah sich als ethnisch deutsch, Frau Konrad hingegen als polnisch an.

Die ethnische Identität äußert sich nicht nur im Sprachgebrauch, sondern bezieht auch die Perspektive der Umstände des Spracherwerbs und die Bedeutung der Sprache(n) für einen Menschen mit ein. Ebenso ist die Frage der Fremdbestimmung und -wahrnehmung relevant (Bracker, 2017, S. 43). Veränderungen in den Lebensumständen, wie vorrangig Migration und Mehrsprachigkeit haben einen immensen Einfluss auf die Identitätskonstruktion und können zu Identitätskonflikten führen.

In beiden Fällen der von mir begleiteten Frauen findet sich eine Migrationssituation, die großen Einfluss auf die Biografie und das Selbstverständnis der Frauen gehabt hat. Auch die subjektiv empfundene Muttersprache erfüllt im Alter und vor allem außerhalb der Heimat eine vielfältige und identitätsstiftende Funktion. Im Fall von Frau Pfeifer nahm die deutsche Sprache eine identitätsstiftende Funktion ein, was in ihrer Lebenswirklichkeit zu keinen weiteren Komplikationen führte, da sie in der deutschsprachigen Umgebung im Einklang mit ihrer eigenen empfundenen ethnischen Identität und besser ausgebauten Sprachumgebung lebte. In ihrem Fall stellt sich nur die Frage, welchen identitären Stellenwert die polnische Sprache einnimmt.

Bei Frau Konrad stellte sich dies anders dar, da sie weniger Möglichkeiten hat, in ihrer polnischen Erstsprache zu kommunizieren und vermehrt auf das Deutsche zurïckgreifen muss, das zugleich jedoch im Zuge der demenziellen Erkrankung zurückging. Es zeigte sich, dass sich die deutsche Sprache bei ihr eher auf rezeptive Fähigkeiten beschränkt, während das Polnische eine essentielle Funktion als Hauptkommunikationsmittel einnahm. In der deutschsprachigen Umgebung des Pflegeheimes war es ihr kaum möglich, Gespräche in ihrer vertrauten Sprache zu führen. Der deutsche Austausch mit den Pflegekräften beschränkte sich entsprechend auf kurze noch mögliche Äußerungen. In den Treffen mit mir war es ihr hingegen möglich, Polnisch zu sprechen und ihre kommunikativen Fähigkeiten 
unter Beweis zu stellen. Ihre subjektiv empfundene ethnische (polnische) Identität war ihr - auch trotz ihrer Erkrankung - klar, und es war ihr möglich, diese nach außen zu tragen. Dabei schien sie sich vielfach der bilingualen und bikulturellen Situation, in der sie sich befand, bewusst. Wie sich dies in späteren Stadien der Demenz gestalten wird, kann man so nicht prognostizieren.

Wirft man nun einen Blick auf die Fremdwahrnehmung, so muss festgehalten werden, dass beide Frauen aus Sicht des Pflegeheimes zur polnischen Gruppe gezählt wurden. Hieran zeigt sich, dass die Fremdwahrnehmung häufig nur einige wenige Faktoren in den Blick nimmt, während andere relevante ausgeblendet werden. Im Fall der beiden Frauen liegt der wesentliche Unterschied in der emotionalen Belegung der beiden Sprachen und damit der Zuordnung der Sprachen als subjektiv empfundene Muttersprache, die ein tiefer emotionaler Bezugspunkt in einem Menschen ist. Als Muttersprache wird meist die ersterworbene Sprache empfunden, die sich ein Mensch in der Regel im familiären und zugleich emotionalen Umfeld aneignet und daher eng mit der Gefühlswelt verknüpft ist. Im Fall von Frau Konrad und Frau Pfeifer ist die zweiterworbene Sprache später im Leben aus funktionellen Gründen hinzugekommen und stellt daher ganz gegensätzliche Bedingungen der Sprachverwendung und der emotionalen Belegung dar. Bei ihnen kann man daher zwischen großen Unterschieden in der Funktionalität und Emotionalität in Bezug auf die polnische und deutsche Sprache sprechen. Frau Pfeifers besser ausgebaute deutsche Sprache war darauf zurückzuführen, dass sie diese als erste erworben, im Elternhaus gesprochen und ihr Leben lang weitergepflegt hat. Die polnische Sprache wurde nur aus funktionellen Gründen erworben. Frau Konrad wurde mit der polnischen Sprache groß, erst im Studium der Zahnmedizin erlernte sie im Rahmen eines Kurses die deutsche Sprache, welche dann bei der Pendelmigration zwischen den Jahren 1989 und 2014 nützlich war.

Aus diesen beiden Fallbeispielen lassen sich mehrere Schlüsse ziehen, die mich noch immer begleiten und in meinem Handeln prägen. So ist hier die Wichtigkeit der Biografiearbeit zu nennen, die ein großes Potenzial hat, die Auseinandersetzung mit der eigenen Identität im Alter zu unterstützen. Sie bietet Menschen die Möglichkeit, eine Auseinandersetzung mit dem bisherigen Leben herbeizuführen und eine Bilanz vor dem nahenden Tod zu ziehen. Durch die Kommunikation kann unser Gegenüber uns etwas über seine Relevanzstrukturen mitteilen, wie er die Wirklichkeit für sich konstruiert (Bracker, 2017, S. 98). Die individuelle Sprachbiografie ist ohne Zweifel ein wichtiger Anhaltspunkt, um über die Identitätswahrnehmung einer Person mehr zu erfahren. Daraus leitet sich die besondere Situation von Menschen mit Migrationserfahrung im Alter ab. Sie stellen eine gesonderte Gruppe dar, da sie gleichzeitig durch alterstypische 
und migrationsspezifische Lebenslagen gekennzeichnet sind. Es kann definitiv behauptet werden, dass ältere Personen mit Migrationshintergrund, welche in deutschen Pflegeheimen wohnen, in der Regel Migrationserfahrungen mit sich bringen, die dazu führen, dass sie sich in besonderer Weise mit ihrem Identitätsund Heimatverständnis auseinandersetzen. Ihre Erfahrungen gelten als Besonderheit, welche zur wachsenden Vielfalt im Alter beitragen und deshalb geschätzt werden sollten. Dies zeigte sich mir bereits in der vertiefenden Betrachtung der Lebensgeschichten von Frau Pfeifer und Frau Konrad. In ihnen zeigte sich ein Bild der demografischen und sozialen Heterogenität älterer Menschen mit polnischem Sprach- und Kulturhintergrund, die zu unterschiedlichen Identitätskonstruktionen führten. Diese Heterogenität hätte ich bei Beginn der Besuche dieser beiden, von außen als polnisch zugeordneten Frauen nicht erwartet.

Nach dem erfolgreichen Abschluss meines Studiums Anfang 2019 kam jener Lebenswunsch stärker an die Oberfläche, nach Polen auszuwandern. Dieser Plan schwebte schon bereits seit meiner Kindheit in meinen Gedanken und nach dem Studium hatte ich glücklicherweise die optimalen Bedingungen dazu, um dieses Vorhaben in die Tat umzusetzen.

Zum Zeitpunkt des Umzuges hatte ich eine Home-Office Tätigkeit, welche ich auch aus Polen ausüben konnte. Dies erleichterte den Start erheblich. Als der Arbeitsvertrag in ein paar Monaten enden sollte und keine Verlängerung geplant war, suchte ich nach neuen beruflichen Möglichkeiten. Eins war dabei für mich wichtig, dass ich einen Beruf ausüben möchte, in welchem ich meine deutschen Sprachkenntnisse einsetzen kann. Und nicht nur das, auch gewisse Kompetenzen, die ich in Deutschland erworben habe, wollte ich mit dem Beruf verbinden. Die Kompetenzen, welche ich im Projekt „UnVergessen“ erwerben durfte, konnten nun ihre Früchte tragen.

Ich sah ein Stellenangebot in einer Pflegevermittlung. Ich bewarb mich. Sofort erkannte ich die Verbindung zum Projekt „UnVergessen“. Es hat sich für mich die Möglichkeit ergeben, einen Probearbeitstag in einer Pflegevermittlung zu machen. Dort bekam ich einen Einblick in die Arbeit eines Koordinators, welcher potenzielle Betreuungskräfte für deutsche Haushalte sucht, Bewerbungsgespräche arrangiert, durchführt und den ganzen Prozess von der Anreise bis zum Aufenthalt in Deutschland und der Abreise begleitet. Die Pflegevermittlung bot mir zuerst eine Anstellung an, änderte jedoch dann die Bedingungen und bot die Möglichkeit an, mit einem eigenen Gewerbe bei ihnen tätig zu werden. Nach kurzen Bedenken sagte ich zu. Am Tag, an dem der Arbeitsvertrag unterschrieben werden sollte, konnten wir uns auf einige bestimmte Punkte im Vertrag nicht einigen, es kam zu keiner Zusammenarbeit. Das Gewerbe war jedoch schon geöffnet und so startete ich meine eigene Pflegevermittlung ,Josmed“. 
Die Altenpflege begleitete mich zwar schon ein Leben lang, aber nun kamen unzählige rechtliche Angelegenheiten auf mich zu, die ersten Monate gestalteten sich sehr schwierig, nach 12-Stunden-Schichten gab es immer noch viel zu tun, und somit kann ich die Worte ,wer selbstständig ist, arbeitet selbst und ständig“ definitiv bestätigen.

Da ein Probearbeitstag nicht gereicht hat, um in alle Arbeitsweisen detailliert hineinzusehen, bestanden die ersten Monate aus kleinen und wichtigen Schritten, um den Bereich rund um eine Pflegevermittlung kennenzulernen. Es begann mit Flyern und Anzeigen im Internet auf sozialen Plattformen, um Josmed nach außen zu tragen und bekannt zu machen. Unzählige Treffen mit Marketingexperten, bis hin zur Ausarbeitung des Firmenlogos. Ich griff auf Kontakte aus dem Projekt zurück, so konnte ich Flyer verteilen, ich schrieb an viele Pflegevermittlungen in Deutschland und Polen, um mein Interesse für eine Zusammenarbeit mitzuteilen. Nach vielen Telefonaten und Mails kam es dann zu einem ersten Treffen zwischen mir und einer anderen Pflegevermittlung aus Polen. Wir unterschrieben einen Kooperationsvertrag, ich vermittele meine Betreuungskräfte an diese weiter. An Betreuungskräfte kam ich durch geschaltete Jobannoncen im Internet und Zeitungen. Ebenfalls kam es zu einer Zusammenarbeit zwischen mir und einer deutschen Pflegevermittlung mit Sitz in Rheinland-Pfalz. Diese stellt Personen aus dem Ausland an und unterschreibt mit ihnen einen deutschen Arbeitsvertrag, diese Form ist in Polen begehrt, da es viele Vorteile mit sich bringt, in dieser Form angestellt zu sein. Die Vorteile zeigen sich im Anspruch auf die deutsche Rente nach einer gewissen Arbeitszeit dank der deutschen Kranken- und Sozialversicherung.

Ich habe im Projekt „UnVergessen“ eine Art der Pflege mitbekommen, welche in Altenpflegeheimen stattfindet. In der Pflegevermittlung vermittle ich Betreuungskräfte, welche bei der zu pflegenden Person im Haushalt leben. Das bedeutet, dass die Betreuungskraft explizit nur für die eine Person zuständig ist. Viele zu betreuende Personen bevorzugen diese Art der Betreuung mehr als die im Altenpflegeheim, wo sich das Personal häufig abwechselt oder aufgrund von Personalmangel eine ausreichende Pflege und Betreuung manchmal nicht umzusetzen ist. Außerdem ist die zu betreuende Person bei sich zu Hause in vertrauter Umgebung, was mit Sicherheit ein anderes Lebensgefühl mit sich bringt.

Da ich nun beide Seiten der Pflege und Betreuung gesehen habe, sehe ich, wie viele Vorteile eine Betreuung im eigenen Zuhause mit sich bringt. Auch hatte ich die Möglichkeit, eine polnischsprachige Betreuungskraft in einen polnischen Betreuungshaushalt zu vermitteln, das Feedback der Familie war sehr positiv, und ich erkannte, dass hier die Kommunikation in der polnischen Sprache ein wichtiger Faktor in der Betreuung war. Die zu betreuende Person hatte so die Gelegenheit, mit ihrer Betreuungskraft in der eigenen Muttersprache zu 
kommunizieren, was so im deutschen Altenpflegeheim in diesem Ausmaß wohl nicht möglich wäre.

Meine Erfahrung aus der Biografiearbeit mit polnischsprachigen Damen im Rahmen des Projektes „UnVergessen“ motiviert mich jeden Tag die Pflegevermittlung zu leiten und diese stetig zu modifizieren und zu verbessern. Ein wichtiger Faktor ist dabei auch die Sprache für mich, die eine enorme Bedeutung in der Beziehung zwischen der zu betreuenden Person und der Betreuungskraft hat.

Im oben erwähnten Fall zeigt sich eine Vermittlung, welche die Kommunikation in der polnischen Sprache möglich macht. Falls eine polnischsprachige Betreuungskraft in eine deutschsprachige Familie vermittelt wird, ist es in diesem Fall auch von großer Bedeutung, dass die Deutschkenntnisse ausreichend sind, um die Pflege und Betreuung durchführen zu können.

Wenn jemand das Bedürfnis hat, so wie ich in der Pflegevermittlung tätig sein zu wollen, sollte er sich vor allem mit vielen rechtlichen Dingen auseinandersetzen. Man muss sich mit Steuern, gesetzlichen Bestimmungen und Versicherungen für die Firma und die Betreuungspersonen auskennen und mit guten Anwälten sprechen. Durchhaltevermögen und Empathie sind meines Erachtens ebenfalls Eigenschaften, die nötig sind, um in dieser Branche Fuß zu fassen und tätig zu sein. Die Konkurrenz ist groß, es gibt viele Pflegevermittlungen sowohl in Deutschland als auch in Polen. Eine Kooperation mit solchen ist immer eine gute Möglichkeit, zusammen etwas Größeres entstehen zu lassen. Und natürlich sollte man diese Arbeit mit Herz machen und nicht vergessen, wer dabei die wichtigste Person ist, um die es dabei wirklich geht: die Person, welche auf die Betreuung und Pflege angewiesen ist.

\section{Am Ende ein Blick in die Zukunft}

Katrin Bente Karl

Nun sind Sie am Ende unserer unterschiedlichen Darstellungen rund um das Projekt UnVergessen angekommen. Ich hoffe, dass Sie einen Eindruck von unseren Tätigkeiten und den Chancen, die sich aus dem Projektdesign ergeben, gewinnen konnten. Wie an vielen Stellen dieser Publikation auf unterschiedliche Weise herausgearbeitet wurde, verstehen wir unsere Projekttätigkeit als eine Verschmelzung vieler Ziele, deren gesellschaftliche und wissenschaftliche Relevanz hier deutlich geworden sein sollte. Die Früchte der bisherigen Tätigkeit sprechen für sich und motivieren mich als Projektleiterin, den Weg weiterzugehen. UnVergessen wird fortgesetzt und sich dabei sicher im Laufe der kommenden Jahre weiter verändern. 
Ebenso bin ich mir sicher, dass neue Erfahrungen auf alle Projektteilnehmer/innen zukommen werden und gehe davon aus, dass auf den unterschiedlichen Ebenen des Projektes weitere Erkenntnisse folgen werden. Damit verbunden ist eine meiner großen Hoffnungen, dass diese wertvollen Erfahrungen genutzt und in darauf aufbauenden Projekten und beruflichen Kontexten umgesetzt werden können. Einige meiner Ideen und konkreten Folgeprojekte mit wissenschaftlicher Ausrichtung habe ich bereits weiter oben (im dritten Teil dieses Buches) dargestellt. An dieser Stelle möchte ich, ergänzend zu den Darstellungen in den beiden vorherigen Beiträgen, aus meiner Sicht die möglichen inner- und außeruniversitären Anknüpfungspunkte und daraus erwachsenden beruflichen Chancen von Studierenden fokussieren. Dies möchte ich anhand von zwei Beispielen herausarbeiten.

Zum einen sehe ich eine besondere Chance und zukünftige Perspektive in der mittlerweile sehr stabilen Vernetzung zweier Institutionen, die beidseitig genutzt und ausgebaut werden kann. Hiermit meine ich die Kontakte zwischen der Universität und den Pflegeeinrichtungen, die auch über das Kernprojekt von UnVergessen hinaus genutzt und unterschiedlichen Zwecken dienen können. In meinen Ausführungen im dritten Teil dieses Buches habe ich bereits ein Beispiel dafür gebracht, wie eine Pflegeeinrichtung die Kontakte zum Projekt nutzte, um in einer konkreten schwierigen Situation Unterstützung zu erhalten (s. die Ausführungen dazu unter dem Kapitel „Das Projekt zu Zeiten von Corona“, gemeint ist hier die Anfrage, für Pflegebedürftige Einkäufe zu tätigen). Die ebenfalls in dem Kapitel geschilderte Aktion Briefe gegen die Einsamkeit ist ein Beispiel, wie das Netzwerk von universitärer Seite genutzt wurde und damit Folgeprojekte ermöglichte. Diese Kontakte liefen dabei über die etablierten Projektkanäle. Daneben bietet sich das Netzwerk aber auch an, um weiteren interessierten Personen und Einrichtungen eine Teilhabe zu ermöglichen. Auch hierfür habe ich bereits an anderer Stelle dieses Buches dargestellt, wie sich das Projekt unter den Pflegeeinrichtungen herumsprach und interessierte Einrichtungen an die Projektleitung mit der Anfrage um Teilnahme herangetreten sind. Ein anderes Beispiel hat sich gerade jüngst aus dem universitären Kontext ergeben. Eine Studierendengruppe meldete sich bei mir und fragte an, ob sie im Kontext eines anderen universitären Projektes das UnVergessen-Netzwerk nutzen dürften. Diese Gruppe belegte ein praktisch orientiertes Modul im Optionalbereich ihres Studiums, in dessen Rahmen sie ein eigenes Projekt mit sozialem Mehrwert entwerfen, planen und umsetzen sollen. Vor dem Hintergrund der pandemiebedingten schwierigen Situation von Pflegebedürftigen in entsprechenden Einrichtungen beschlossen sie, in diesem Bereich ihr Projekt anzusiedeln. Bei der anschließenden Recherche stieBen sie auf die Aktion Briefe gegen die Einsamkeit und nahmen Kontakt zu mir 
auf. Nach einem ersten Austausch stand schnell fest, dass die Projektidee in schönem Einklang zu den Ideen und Zielen von UnVergessen steht, weswegen dieser Studierendengruppe sehr gerne der Kontakt zu interessierten Kooperationspartnern in den Einrichtungen vermittelt wurde. Die Ausrichtung dieses studentischen Projektes mit Namen ESADA, als Akronym aus den Namen der Student/innen (Elena Schneider, Sophie Lohkamp, Angin Kuriewicz, Daniel Ruhmöller, Anna Petcheeva) entstanden und zum Motto „Eine Stimme Aus Dem Altenheim“ ausgeweitet, ist dabei, dass mithilfe von Leitfragen Berichte und Erfahrungen von Pflegebedürftigen in schriftlicher Form gesammelt und schließlich in Form einer Ausstellung oder eines anderen geeigneten Mediums einer interessierten Öffentlichkeit präsentiert werden. Damit möchten die Studierenden dieser vulnerablen Gruppe eine Stimme verleihen. Über das UnVergessen-Netzwerk konnten die Studierenden Kontakt zu ausgewählten Kooperationspartnern herstellen und dort ihre Idee vortragen. Erfreulicherweise kam in allen Fällen eine positive Reaktion bei der Erwähnung der Nähe zu UnVergessen zurück und es fanden sich interessierte Einrichtungen, die wiederum an interessierte Bewohner/innen vermittelten. Zum Zeitpunkt der Drucklegung dieses Buches befindet sich das Projekt ESADA in der Phase, dass die Erhebung der schriftlichen Daten kurz vor der Durchführung steht. Auf die Berichte und Erfahrungen der Gruppe bin ich sehr gespannt und hoffe, dass auch dieses Engagement zu mehr Sichtbarkeit und Verständnis für die Gruppe der Pflegebedürftigen führt.

Damit sollte ersichtlich geworden sein, dass die Kontakte nicht exklusiv Teilnehmer/innen am Projekt UnVergessen zur Verfügung stehen, sondern zum gegenseitigen Nutzen eingesetzt und ausgebaut werden. Dies setzt voraus, dass die Tätigkeiten rund um UnVergessen einen gewissen Bekanntheitsgrad haben, zugleich erfordert es eine Offenheit gegenüber neuen Ideen und Menschen und nicht zuletzt auch gegenseitigen Respekt.

Der zweite Bereich, den ich hier konkretisieren will, sind Anknüpfungsmöglichkeiten in das berufliche Leben. Hier beobachte ich seit zwei Jahren, dass unter den studentischen Teilnehmer/innen von UnVergessen eine wachsende Anzahl an Personen zu finden ist, die Vorerfahrungen im Pflegekontext aufweisen. Sei es durch eine vorherige Ausbildung zur Pflegekraft, eine parallele Arbeit in einer Pflegeeinrichtung - auch der teilstationären Pflege - oder andere berufliche Querverbindungen. Diese Teilnehmer/innen bringen ihre Erfahrungen in der Pflege auf eine sehr bereichernde Art in das Projekt ein und können diese um die sprachlichen Facetten und den Schwerpunkt auf die Mehrsprachigkeit vertiefen. Auf diese Weise finden sich spezialisierte und sensibilisierte Personen, die ihre vielfältigen Erfahrungen in die Praxis umsetzen können. Sei es durch ihre direkte Arbeit in den Pflegeeinrichtungen oder durch die Entwicklung von 
weiterführenden Ideen. Dabei steht seit ca. zwei Jahren die bislang noch etwas vage Idee im Raum, ein Start-Up-Unternehmen zu gründen, das zum Ziel hat, Wohn- und Pflegekonzepte im Ruhrgebiet ins Leben zu rufen, die an die speziellen Bedürfnisse mehrsprachiger Pflegebedürftiger angepasst sind. Dafür gibt es einige Vorbilder, die eine ähnliche Fokussierung bereits seit mehreren Jahren leben. Speziell für unseren Sprachkontext sei hier auf eine seit dem Jahr 2006 in Köln ansässige ambulant betreute Pflegewohnung für russischsprachige Menschen mit dem Namen Nascha Kwartihra (deutsch: ,Unsere Wohnung') verwiesen. Dort können acht an Demenz erkrankte russischsprachige Personen betreut und gepflegt werden (vgl. https://wohnkonzepte-schneider.de/nascha-kwartihra/). Für den türkischen Sprachraum finden sich ebenso Vorbilder, wie bspw. eine aus einem Projekt hervorgegangene türkisch-muslimische Wohngemeinschaft für betreuungs- und pflegebedürftige Frauen in Sindelfingen (für mehr Informationen s. https://www.lrabb.de/site/LRA-BB-Desktop/get/params_E-1225952024/160 67847/Projekt-Dokumentation\%20YASAM\%20EVI.pdf) oder das in Hamburg ansässige ambulant betreute Wohnen für Menschen türkischer Herkunft mit Demenz, über das u. a. von der Deutschen Alzheimer Gesellschaft im Jahr 2015 berichtet wurde (vgl. hierzu: https:/www.deutsche-alzheimer.de/unser-service/ archiv-alzheimer-info/ambulant-betreutes-wohnen-fuer-menschen-tuerkischerherkunft-mit-demenz.html). Es gibt bundesweit weitere ähnlich ausgerichtete Wohnprojekte und - wie auch bereits an anderer Stelle ausgeführt - unterschiedliche Ansätze, verschiedene Sprachen und Kulturen in die unterschiedlichen Formen der Pflege zu integrieren. Diese sehe ich als Zeichen für ein wachsendes Verständnis und Diversität in Pflegeeinrichtungen und zugleich als Zeichen dessen, dass es jetzt an der Zeit ist, diesen Prozess fortzusetzen und zu vertiefen. Hier sehe ich Studierende mit mehrsprachigem Hintergrund und zugleich mit Einblicken und Erfahrungen in der Pflege als wichtige Multiplikatoren und mögliche Initiatoren für die Umsetzung kultur- und sprachsensibler Betreuungsformen. Diese Idee wurde im Laufe des Durchganges 2019/2020 immer wieder thematisiert und meine Vision ist, dass sich interessierte Studierende über das UnVergessen-Netzwerk finden und daran weiterarbeiten. Dies erscheint speziell vor dem Hintergrund der Ausrichtung der Ruhr-Universität Bochum besonders Erfolg versprechend, da dort im Laufe der letzten Jahre diverse Programme zur Unterstützung im Bereich der Gründung initiiert wurden, die in besonderer Weise in der WORLDFACTORY zusammenlaufen. Beratung und Unterstützung von dieser Seite wäre damit zeitgleich auch möglich.

Bei der Entwicklung und Umsetzung solcher Ideen sehe ich UnVergessen, das Netzwerk und mich als Impulsgeberin, Wegbegleiterin und Partnerin. Meine Hoffnung ist, dass sich auf diese Weise viele spannende Ideen entwickeln und sich 
engagierte und mutige Menschen finden, diese umzusetzen und mit Leben zu füllen.

\section{Literatur}

Bracker, P. (2017). Die Entstehung ethnischer Identität bei Menschen mit türkischem Migrationshintergrund. In W. Grießhaber \& J. Rehbein (Hrsg.) Mehrsprachigkeit (Bd. 44). Waxmann Verlag.

Krappmann, L. (2000). Soziologische Dimensionen der Identität: Strukturelle Bedingungen für die Teilnahme an Interaktionsprozessen. Klett-Cotta.

Tepper, M. (2012). Was bleibt ist ein Mensch! https://pflegendeangehoerige.wordpress.com/ 2012/12/07/was-bleibt-ist-ein-mensch-2/.

Open Access Dieses Kapitel wird unter der Creative Commons Namensnennung 4.0 International Lizenz (http://creativecommons.org/licenses/by/4.0/deed.de) veröffentlicht, welche die Nutzung, Vervielfältigung, Bearbeitung, Verbreitung und Wiedergabe in jeglichem Medium und Format erlaubt, sofern Sie den/die ursprünglichen Autor(en) und die Quelle ordnungsgemäß nennen, einen Link zur Creative Commons Lizenz beifügen und angeben, ob Änderungen vorgenommen wurden.

Die in diesem Kapitel enthaltenen Bilder und sonstiges Drittmaterial unterliegen ebenfalls der genannten Creative Commons Lizenz, sofern sich aus der Abbildungslegende nichts anderes ergibt. Sofern das betreffende Material nicht unter der genannten Creative Commons Lizenz steht und die betreffende Handlung nicht nach gesetzlichen Vorschriften erlaubt ist, ist für die oben aufgeführten Weiterverwendungen des Materials die Einwilligung des jeweiligen Rechteinhabers einzuholen. 